\title{
Motoric Cognitive Risk Syndrome: A Risk Factor for Cognitive Impairment and Dementia in Different Populations
}

\author{
Zeev Meiner ${ }^{1}$, Emmeline Ayers ${ }^{2}$, Joe Verghese ${ }^{2,3}$ \\ ${ }^{1}$ Department of Physical Medicine and Rehabilitation, Hadassah Mount Scopus, Jerusalem, Israel \\ ${ }^{2}$ Department of Neurology, Albert Einstein College of Medicine, Bronx, NY, USA \\ ${ }^{3}$ Department of Medicine, Albert Einstein College of Medicine, Bronx, NY, USA
}

\author{
Corresponding Author: \\ Joe Verghese, MBBS \\ Department of Neurology, Albert \\ Einstein College of Medicine, 1225 \\ Morris Park Avenue, Bronx, NY 10461, \\ USA \\ E-mail: joe.verghese@einsteinmed.org \\ ORCID: \\ https://orcid.org/0000-0003-4252-2547
}

Received: January 10, 2020

Revised: February 17, 2020

Accepted: February 18, 2020

\begin{abstract}
Changes in gait, especially decreased gait velocity, may be a harbinger of cognitive decline in aging. Motoric cognitive risk syndrome (MCR), a pre-dementia syndrome combining slow gait and cognitive complaints, is a powerful clinical tool used to identify older adults at a high risk of developing dementia. The mean prevalence of MCR worldwide, including in a Korean cohort, was around $10 \%$. The reported risk factors for incident MCR include older age, low education, cardiovascular disease, obesity, physical inactivity, and depression. In addition to dementia, MCR is also a risk factor for other age-related adverse conditions such as falls, disability, frailty, and mortality. The use of MCR has advantages over other pre-dementia syndromes in being much simpler to implement and requires fewer resources. Identification of mechanisms responsible for MCR may help in developing interventions to reduce the growing burden of dementia and disability worldwide.
\end{abstract}

Key Words: Motoric cognitive risk syndrome, Gait, Cognitive impairment, Dementia

\section{INTRODUCTION}

The population worldwide is aging rapidly. The percentage of people above 65 years of age is the most rapidly growing segment of the population and is estimated to nearly double between 2019 and 2050. ${ }^{1)}$ Therefore, age-related diseases are becoming an important burden on people's lives and countries' economies. Dementia and cognitive disorders are common among the aging population and play an important role in increasing morbidity and reducing the quality of life of older adults. ${ }^{2)}$ Over 50 million people were estimated to be living with dementia globally in 2019 and this number is expected to increase to 152 million by 2050 . The current estimated annual cost of dementia is US \$1 trillion globally, a figure set to double by 2030. Gait disturbances are also common with aging and are associated with increased morbidity and mortality. ${ }^{3)}$

Given this global burden associated with aging, there is a press- ing need to identify older adults at risk for dementia to institute preventive interventions, make healthcare decisions, and consider advanced planning decisions. Cognitive decline and dementia have been implicated in gait dysfunction through disturbances in top-down brain control mechanisms. ${ }^{4)}$ Emerging evidence supports gait dysfunction as a harbinger of dementia; this association could be a novel method to assess dementia risk. ${ }^{5)}$ Motoric cognitive risk syndrome (MCR) is a pre-dementia condition combining motor signs (slow gait) and cognitive symptoms (self-reported cognitive complaints). ${ }^{6}$ Studies across the globe have shown that the presence of MCR increases the risk of dementia as well as other age-related adverse outcomes. More recent studies have also uncovered links between MCR and genetic, metabolic, and imaging factors. ${ }^{7)}$

This review discusses the epidemiology of MCR, its role in increasing the risks of dementia and other geriatric outcomes, and recent biological discoveries regarding its pathophysiology. A sys- 
tematic search was conducted using the Medical Subject Heading terms "slow gait", "subjective cognitive impairment", "subjective cognitive decline", and "motoric cognitive risk syndrome".

\section{GAIT SPEED AND COGNITIVE DECLINE IN GENERAL}

Gait is a complex and multifactorial process in terms of its underlying central and peripheral neural control mechanisms. Similar brain regions control both gait and cognitive functions, particularly frontal and prefrontal lobe-related networks. ${ }^{8}$ These brain areas are responsible for mediating executive functions (EFs), a variety of higher cognitive processes that integrate information from many cortical sensory systems and modulate and produce effective and goal-directed actions and behavior. ${ }^{9,10)}$ The aging process is accompanied by atrophy of many of these brain regions, causing both cognitive and gait decline. ${ }^{11-13)}$ Changes in the aging brain include atrophy of the frontal and temporal areas and the occurrence of periventricular white matter lesions. Abnormal gait is a prominent feature in neurodegenerative diseases, especially those that affect mainly the frontal lobes. ${ }^{14,15)}$ Pilot intervention trials to enhance EF either by cognitive training or brain stimulation have shown improvements in gait velocity. ${ }^{16,17)}$

Slow gait speed may be the first sign of degenerative or non-degenerative brain pathologies and may manifest before other cognitive symptoms. Clinical gait disturbances in older adults may be due to neurological, muscular, or arthritic etiologies as well as combinations of these factors. Neurological gait abnormalities were reported to predict the incidence of non-Alzheimer's dementia in the Bronx Aging Study. ${ }^{18)}$ Velocity is the most widely used quantitative performance index of gait; however, other gait variables such as stride length, cadence, swing and stance time, and symmetry obtained from quantitative gait assessments are also used to evaluate gait quality. ${ }^{19)}$ Growing evidence suggests that not only a decline in gait speed predicts dementia but also that a decline in gait speed may precede the decline in cognitive performance in dementia. ${ }^{720)}$ These observations suggest that clinical or quantitative gait disturbances may be used to identify people at risk to develop dementia.

\section{MCR DIAGNOSIS ACROSS POPULATIONS}

In 2013, Verghese et al. ${ }^{21)}$ introduced the concept of MCR to describe people who are still cognitively intact but with cognitive complaints and slowing of gait, who are at higher risk of developing dementia. The criteria for MCR in this initial study were built on those for mild cognitive impairment syndrome (MCI), ${ }^{22)}$ and included the presence of subjective (self-reported) cognitive com- plaints measured by a structured questionnaire or clinicians' interview, slowness of gait defined as one standard deviation (SD) below age- and sex-specific gait speed mean values established in the same population, independence in activities of daily living, and absence of dementia. The main criterion distinguishing MCR from MCI was substituting a slow gait criterion for objective impairment on a cognitive test in MCI. Out of the 997 community-residing individuals aged 70 years and older participating in the Bronxbased Einstein Aging Study, 7\% met this operational definition of MCR. Over a follow-up period of 36.9 months, those diagnosed with MCR at their baseline visit had a higher risk of developing dementia, especially vascular dementia, compared to those without MCR at baseline. ${ }^{19)}$ Since then, the prevalence of MCR has been examined in many other cohorts and populations worldwide and found to vary between $2 \%$ and $27 \%$ (Table 1). ${ }^{21,23-43)}$ These studies differed in the way MCR and the reported MCR risk factors in different populations. The differences in estimated MCR prevalence may be attributed to the way MCR criteria are operationalized in studies as well as the different populations recruited in previous studies. Cognitive tests are not required to diagnose MCR, which increases its clinical utility. The assessment for subjective cognitive complaints was performed using different methods in different cohorts, such as the 15-item Consortium to Establish a Registry for Alzheimer's Disease (CERAD) questionnaire, ${ }^{44)}$ one or two incorrect responses on the Short Portable Mental Status Questionnaire (SPMSQ) ${ }^{45)}$ the memory item from the 15 -item Geriatric Depression Scale, ${ }^{46)}$ the eight-item informant interview (AD8), ${ }^{47)}$ or the Clinical Dementia Rating scale. ${ }^{48)}$ In other studies, positive responses from participants to questions such as "Do you have trouble remembering?" or "Is your memory worse than 10 years ago?" were sufficient. Even a referral to a memory clinic has been used to assess for the MCR subjective cognitive complaint criterion. ${ }^{41,42)}$ This heterogeneity in subjective cognitive complaint ascertainment may lead to differences in prevalence estimates of MCR as well as variability between studies in the cognitive impairment of individuals diagnosed with MCR. Subjective cognitive complaints related to the early stages of dementia may also be expressed differently in different cultures and parts of the world. As subjective cognitive complaints are key criteria to diagnose $\mathrm{MCI}$ and dementia, issues regarding the specificity of this criterion are not unique to MCRs.

All MCR studies have used normal walking gait velocity to evaluate gait slowness; however, methods have varied across studies with assessments done either by instrumented walkways such as the GAITRite system ${ }^{49)}$ (CIR Systems Inc., Sparta, NJ, USA) or by timing participants' walk at normal pace using a stopwatch over a fixed distance. The distances used have also varied from $2.44 \mathrm{~m}(8$ feet) to $9.70 \mathrm{~m}$ (20 feet), which may also influence slow gait deter- 


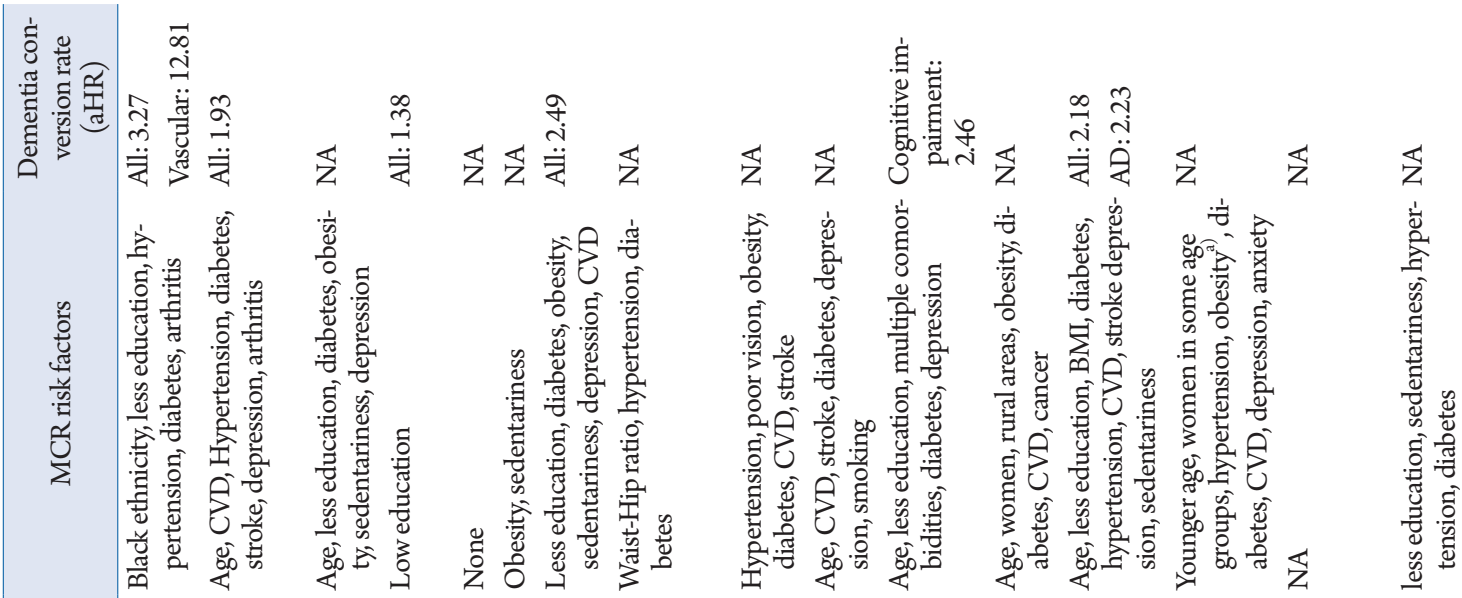

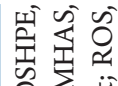

赵苛

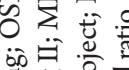

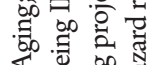

$\exists$ 近

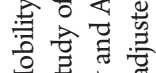

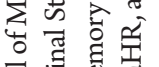

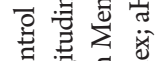

记

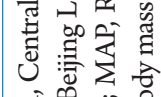

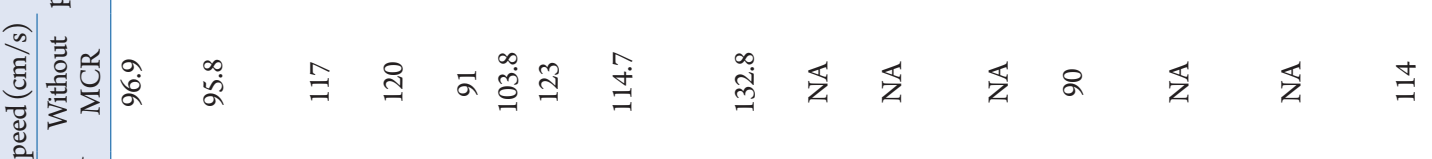

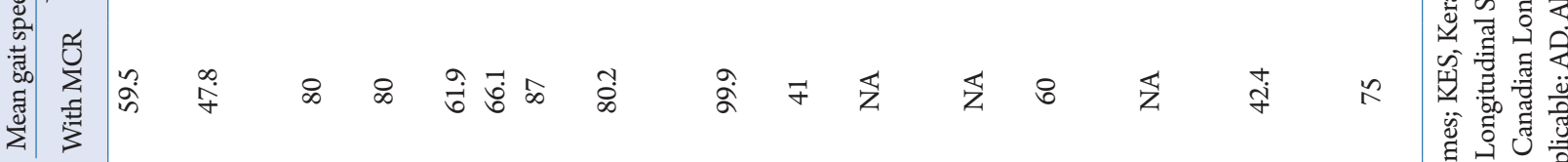

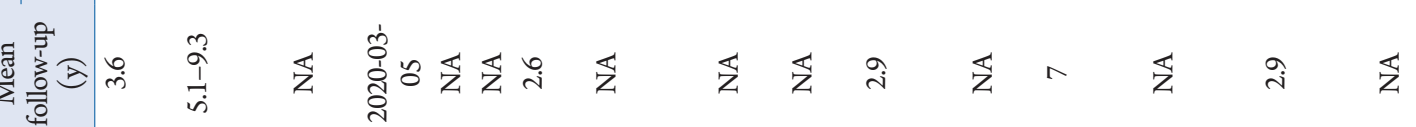

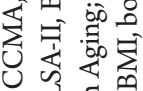

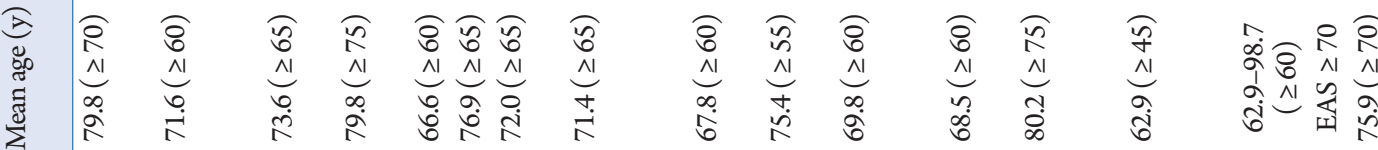

窟品

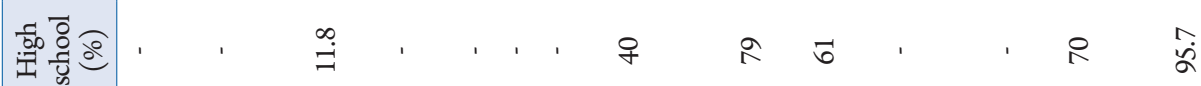

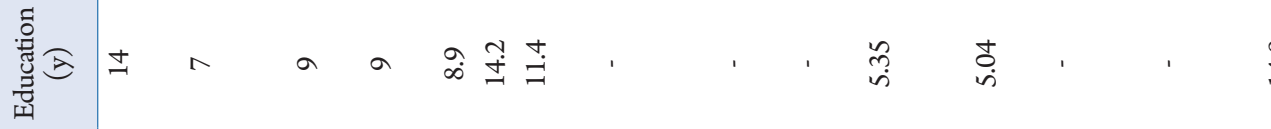

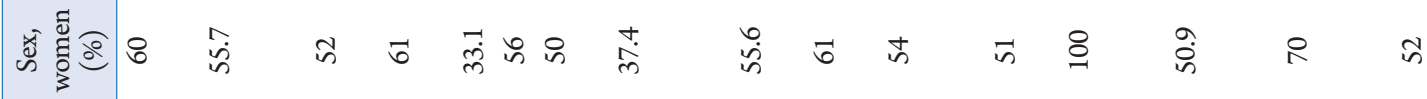

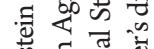

落

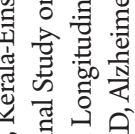

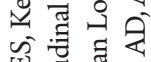

灵营

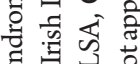

否矛苟

焉焉

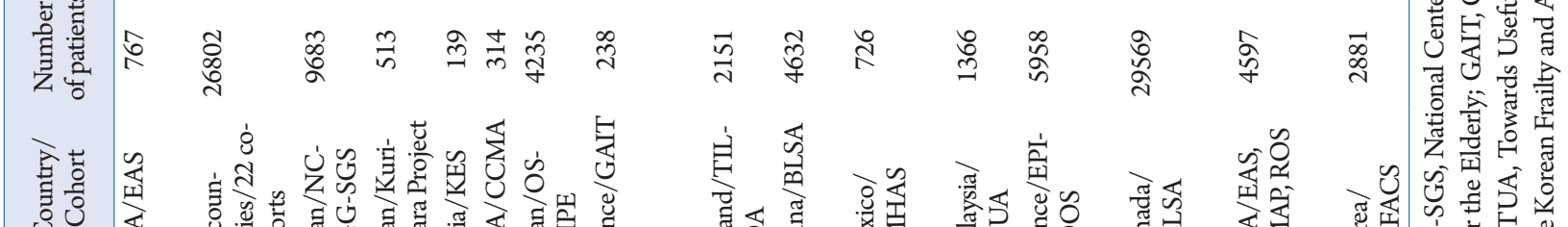

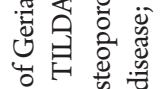

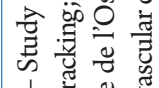

ถิำ

ֻேं

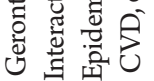


mination. Differences in measuring methods and in population characteristics explain why the cutoff velocity for the diagnosis of MCR is so different between studies (Table 1). Although all studies defined slow gait as walking speed $1 \mathrm{SD}$ below age- and sex-specific means individualized to each cohort, the mean velocity for each cohort varies significantly. The mean velocity for the diagnosis of MCR varies between $41 \mathrm{~cm} / \mathrm{s}^{34)}$ and $99.9 \mathrm{~cm} / \mathrm{s}^{33)}$ Age and sex are important factors in determining gait velocity; therefore, many studies calculated specific means for different age groups to reduce heterogeneity. Although a universal single cutoff velocity for determining slow gait criterion may add clinical utility to the MCR definition, it may not be practical given the variability in gait velocity across age groups, sexes, and populations. ${ }^{50)}$ Other motoric tests such as the Five-Times-Sit-to-Stand Test (FSTT) were less specific to diagnose MCR when used as a substitute for gait velocity. ${ }^{51,52)}$ Other quantitative gait variables have been used to diagnose MCR subtypes and were shown to identify older adults with different cognitive trajectories ${ }^{29)}$ and risk factors ${ }^{52)}$ than the classical MCR definition. However, assessment for these subtypes requires access to an instrumented walkway, which limits its use in many clinical settings.

The third component of MCR is independence in activities of daily living. This component was examined in published studies using either a structured questionnaire developed to assess function in community-residing older adults ${ }^{53)}$ or by study clinician interviews. The multi-country MCR prevalence study used mobility disability for this criterion as information on activities of daily living were not available in all of the 22 included cohorts. ${ }^{23)}$ The fourth component of the syndrome is the absence of dementia. This was assessed either using known clinical criteria for dementia, such as those in the Diagnostic and Statistical Manual of Mental Disorders, 4th edition (DSM-IV), ${ }^{54)}$ and diagnosed at consensus case conferences, through participant or informant report of physician-diagnosed dementia, or by applying established cut-off scores on cognitive tests such as the Mini-Mental State Examination (MMSE) ${ }^{55)}$ These criteria vary considerably across studies and may result in the inclusion of individuals with different levels of cognitive disturbance.

Study populations have varied across MCR studies. While most studies were restricted to older populations, some also included younger people. Most studies used an entry age of 60,65 , or 70 years. Two studies included only adults 75 years and above, ${ }^{26,37)}$ while two other studies used an entry criterion of 45 years. ${ }^{34,40)}$ This difference in the age of entry affects the determination of slow gait and other parameters related to MCR prevalence and risk factors. The prevalence of women in the studies varies between $33 \%$ and $70 \%$, with one study including only women. ${ }^{37)}$ Recruitment methods also increased the variability of study populations in previous reports. A multi-country prevalence study included data from 22 cohorts, ${ }^{23)} 16$ of which were community-based, 4 were recruited participants from memory clinics, and 2 were recruited from both clinics and the community. The highest prevalence of MCR was reported in the Indian and French cohorts, which were memory clinic-based populations. ${ }^{23)}$ MCR studies have included data from cohorts from different countries and ethnic groups. In one US-based study that included both African American and Caucasian populations, the prevalence of MCR was higher among African American participant. ${ }^{21)}$ MCR studies have also been conducted in individuals with low income and low education levels. ${ }^{23,28,35,36)}$ The MCR definition has shown relatively stable prevalence estimates in studies with various populations and variable education and socioeconomic levels, supporting its role as a practical and reliable clinical assay for dementia risk worldwide.

\section{MCR Risk Factors across Populations}

The risk factors for MCR include age, sex, level of education, obesity, physical inactivity and sedentariness, depressive mood, and cardiovascular disease. Most studies reported that advanced age increased the risk of MCR, ${ }^{23,25,34-37)}$ similar to dementia. For instance, in a multi-country MCR study that included over 26,000 participants, ${ }^{23)}$ the MCR prevalence was $10.6 \%$ in the group aged 75 years and older as compared to $8.9 \%$ in the group aged $60-74$ years. However, MCR studies from Ireland and Korea reported that the prevalence of MCR did not increase with advancing age and was similar across age groups. ${ }^{33,43)}$ In one study from Cana$\mathrm{da},{ }^{37)}$ the risk of MCR was higher in the younger age groups (4555 years) compared to that in more advanced age groups. However, this was the only community-based study to include such a young age group. The next-highest prevalence of MCR in this study was that in the older group of 75 years and above. This finding of increased MCR prevalence with age is consistent with the reported higher prevalence of cognitive complaints and dementia with increasing age.

Most studies did not find any difference in MCR prevalence between sexes. However, some studies reported a higher rate of MCR among women. In a prevalence study from Malaysia that included 1,366 participants, the prevalence rate of MCR was 5\% among women and $1.8 \%$ among men. ${ }^{36)}$ The authors attributed this finding to the higher prevalence of frailty among older Malaysian women compared to that in men. A Canadian study ${ }^{37)}$ reported a higher MCR prevalence in women aged 45-54 and 65-74 years but with opposite results in the other age groups, a finding with unclear interpretation.

Most, but not all MCR studies found that lower levels of educa- 
tion are a risk factor for MCR. $23,25,30,35,37,43)$ Most studies used years of schooling to measure education, which may not be a perfect measure. In a Canadian study with 29,569 participants, lower education level was a risk factor for MCR only in the 55-64 and 65-74 years age groups and not in the youngest or the oldest groups. ${ }^{40)}$ In a multi-country study including more than 26,000 participants, a higher level of education ( $>12$ school years) was associated with a lower prevalence of MCR. ${ }^{23)}$ The best explanation is that, similar to MCI and dementia, higher levels of education are also a protective factor for MCR, perhaps by increasing brain cognitive reserves. ${ }^{56)}$

Similar to studies of MCI and dementia, ${ }^{57,58)}$ most studies reported sedentary lifestyle to be a risk factor for MCR. ${ }^{25,29,30,37,43)}$ The methods used to determine levels of physical activity varied between studies, with some studies adopting a structured questionnaire and others using a simple question regarding regular participation in a sport or leisure physical activity (i.e., at least 1 hour a week for the past month). Sedentariness was also examined by self-reported difficulty in walking less than a quarter mile or negotiating stairs. ${ }^{59)}$ The TILDA study based in Ireland ${ }^{33)}$ reported no significant correlation between physical activity level and MCR prevalence; however, participants in this study were more likely to be cognitively healthy and with high physical functioning. The protective effect of physical activity in MCR, like in MCI and dementia, can be explained by several mechanisms such as the positive effects of physical exercise on cardiovascular risk factors including hypertension, insulin resistance, and high cholesterol levels as well as other biological mechanisms such as enhanced immune system function, anti-inflammatory properties, and increased neurotrophic factors. ${ }^{60)}$

One notable risk factor of MCR was obesity, defined either by body mass index (BMI) or waist-to-hip ratio. ${ }^{25,29,30,33,36,37,40)}$ Most MCR studies did not evaluate body composition. In two studies from China and Korea, obesity was not correlated with MCR, suggesting possible ethnic differences in this MCR risk factor. ${ }^{34,43}$

Cardiovascular diseases and cardiovascular risk factors such as hypertension and diabetes increase MCR risk, similar to the increased risk reported for these risk factors with dementia and MCI. ${ }^{61-63)}$ Diabetes was a risk factor in almost all studies. ${ }^{34)}$ Hypertension was a risk factor in most, but not all, studies. ${ }^{25,34,36)}$ Ischemic heart disease was also a risk factor in the majority of studies. $^{23,30,33,34,36,37,40)}$ Smoking was identified as a risk factor for MCR in one study from China ${ }^{32)}$ but not in other studies in which it was documented. ${ }^{25,30,36)}$ Alcohol consumption was not found to be a risk factor for MCR in any of the studies.

Polypharmacy is a known risk factor for frailty and cognitive decline in old age. ${ }^{(4)}$ Several studies have examined the relationship between the number of medications prescribed and the occurrence of MCR; $;^{25,30,33,37-39,42)}$ most studies reported that increased numbers of medications were associated with MCR. The mean number of medications taken by participants with MCR varied between 2.3 and 6.6. Using a widely accepted definition of polypharmacy as five or more medications daily, two studies showed that polypharmacy was a risk factor for MCR. $33,37,38)$

Depression and anxiety are strongly associated with MCR. This association was found in large-scale studies including a multi-country study and the Canadian CLSA study. ${ }^{23,40)}$ The latter study observed this association in all age groups. The use of anti-depressive medications was also associated with MCR. ${ }^{33)}$ Depression in old age is related to cognitive decline and dementia and may be an early manifestation of dementia due to neurodegenerative or vascular brain diseases. ${ }^{65}$ Other significant risk factors for MCR examined in individual studies were arthritis, ${ }^{21,23)}$ poor vision, ${ }^{33)}$ and living in rural areas. ${ }^{36)}$

\section{MCR as a Risk Factor for Cognitive Decline and Dementia}

A recent meta-analysis by Sekhon et al. ${ }^{66)}$ including seven studies evaluated the relationship between MCR and the development of cognitive impairment and dementia and examined the relationship between MCR and the development of either dementia or cognitive impairment. In general, MCR was associated with increased risks of incident cognitive impairment (adjusted hazard ratio $[\mathrm{aHR}]=1.70)$ and dementia $(\mathrm{aHR}=2.50)$. This meta-analysis included four studies that examined the association between MCR and the incidence of dementia. ${ }^{21,23,26,30)}$ Since then, another study from France reported an association between MCR and dementia. ${ }^{38)} \mathrm{MCR}$ has been shown to have incremental predictive validity for dementia over its cognitive (cognitive complaints) and motoric (slow gait) components. ${ }^{21}$

There is heterogeneity in terms of study populations, follow-up time, and criteria employed for the diagnosis of MCR and cognitive disorders among studies that have examined the predictive validity of MCR for cognitive decline. In a homogenous cohort from the US, ${ }^{21)}$ the aHR to develop dementia overall in participants with MCR was 3.27; however, MCR did not predict Alzheimer disease $(\mathrm{AD})$ but did strongly predict vascular dementia $(\mathrm{aHR}=$ 12.81). This finding was consistent with that of a previous study that showed slow gait to be a predictor of vascular dementia and not $\mathrm{AD}^{21)}$ In contrast, a combined analysis of four cohorts that included 4,812 participants with longitudinal data reported MCR to be a risk factor for dementia overall including $\mathrm{AD}(\mathrm{aHR}=$ 1.93). ${ }^{23)}$ In a study from Japan including 4,235 participants, ${ }^{30)}$ over a mean follow-up of 2.6 years, the aHR to develop dementia overall in participants with MCR at baseline was 2.49; however, the de- 
mentia subtypes were not specified. In another small study from Japan including 299 participants with a follow-up of 3-5 years, the odds ratio of conversion to dementia overall was 1.38 in the MCR group compared to the non-MCR group. A recent study of 651 French women followed up to 7 years ${ }^{38)}$ reported a nearly two-fold increased risk of incident dementia in the MCR group than that in the non-MCR group (41.9\% vs. $23.3 \%$ ). MCR was positively associated with the incidence of overall dementia and $\mathrm{AD}$ but not with non- $\mathrm{AD}$ dementia incidence. This study included only women; therefore, the results may not apply to the general older population.

A recent multicenter study including 610 older adults with MCR from three US-based cohorts followed over a mean of 2.9 years investigated which components of the MCR syndrome predicted transition to dementia. ${ }^{42)}$ The cognitive components measured by a cognitive complaint severity index, logical memory test, ${ }^{67)}$ and $\mathrm{MMSE}^{55)}$ — predicted the transition from MCR to dementia, whereas the motoric component of MCR (gait velocity) did not. This finding may be attributed to the fact that the cognitive complaints and tests in MCR patients may better correlate with the worsening of dementia-related pathology or spread of pathology into brain areas responsible for other non-motor behaviors and cognitive impairments associated with dementia. An alternative explanation might be that, since this study included only individuals with MCR with a restricted range of gait velocities, slower gait was not a predictor of dementia. Finally, since memory-related questions were used for the diagnosis of MCR and the incident dementia cases included a high proportion of $\mathrm{AD}$ patients, cognitive complaints but not gait velocity predicted dementia in this analysis.

Several studies evaluated the association between MCR and other pre-dementia syndromes such as MCI. ${ }^{21,23,31)} \mathrm{MCI}$ diagnosis is based on subjective cognitive complaints (as in MCR), objective cognitive impairment in the memory or non-memory domains assessed by neuropsychological tests, and without impairment of activities of daily living or dementia. ${ }^{68)}$ The co-occurrence of MCI among MCR was $54 \%, 47 \%$, or $39 \%$ in different studies, respectively. $^{21,23,31)}$ The combination of MCR and MCI was associated with lower cognitive performance compared to that in individuals with MCR but without MCI. ${ }^{31)}$ While there is overlap between MCR and MCI cases, MCR syndrome still statistically predicted the risk of dementia in previous studies after accounting for MCI cases or excluding individuals who met criteria before or simultaneously with MCR. These observations emphasize the importance of diagnosing both pre-dementia syndromes to identify all individuals at risk. MCR should be seen as a complementary rather than an alternative approach to MCI.

Six studies evaluated the association between MCR and cogni- tive performance, with mixed results. ${ }^{23,26,29,31,33,43)}$ These studies assessed several domains of cognitive function, including global cognitive functions, memory, EF, processing speed, attention, visuospatial abilities, and language. In most studies, MCR was negatively associated with global cognitive performance and $\mathrm{EF}$, supporting the hypothesis that frontal lobe dysfunction is involved in both gait and EF control. ${ }^{9}$ However, one study did not show an association with $\mathrm{EF}$ but found that the MCR group performed worse on measures of global cognition, memory, and sustained attention. ${ }^{33)}$ The authors attributed this finding to the larger size of their sample and the specific characteristics of the population in this cohort (TILDA). The relationship between memory functions and MCR is conflicting, with several studies reporting lower performance in memory tests in $\mathrm{MCR},{ }^{23,31,33)}$ while others did not find such a correlation. ${ }^{29,43)}$ One study reported an association between language difficulties and MCR; ${ }^{29)}$ however, other studies did not test this domain.

\section{MCR as a Risk Factor for Other Geriatric Outcomes}

MCR is reportedly a risk factor not only for dementia but also for other adverse conditions in older adults such as falls, disability, frailty, and mortality. ${ }^{69)}$ Falls are an important medical problem in the older population. An estimated $32 \%-42 \%$ of all people above 70 years of age will fall every year. ${ }^{70)}$ Falls in older adults are associated with complications including fractures, surgery, and hospitalization and are related to increased disability and mortality. ${ }^{71)}$ Maintaining balance and preventing falls is a complicated function that requires efficient integration of motoric, cognitive, and psychological functions. Individuals with MCR have a combination of cognitive impairment, mainly in EF, and motor disturbances that place them at high risk for falls. Several studies have investigated this association. Callisaya et al. ${ }^{72)}$ examined 6,204 participants from five large cohorts across three countries and revealed that $33.9 \%$ of subjects with MCR reported a fall over follow-up of 12 months, resulting in a pooled relative risk (RR) of MCR for any falls of 1.44; this association reduced in strength but persisted after adjusting for previous falls $(\mathrm{RR}=1.37)$. As for dementia outcomes, ${ }^{73)}$ MCR had incremental predictive validity for falls over its cognitive and motoric components. In a study of 2,569 French women, MCR increased the risk for any fall $(\mathrm{aHR}=1,22)$, recurrent falls $(\mathrm{aHR}=1.46)$, and falls complicated by hip fracture $\left.(\mathrm{aHR}=2.54){ }^{37}\right)$

Few studies have examined the association between MCR and disability and frailty among older adults. In a study of 4,235 Japanese older adults (mean age, 72 years), MCR was associated with an increased risk of disability, defined as certification by long-term care insurance, with an aHR of $1.69 .^{30)}$ Frailty is a multidimension- 
al construct associated with low physiologic reserves and increased vulnerability to adverse outcomes such as disability and death. ${ }^{74)}$ In a study of 641 adults aged 65 years and above, higher frailty at baseline, as assessed using a cumulative deficit index, increased the risk of developing incident MCR even after accounting for several confounders, suggesting shared mechanisms between these two syndromes. $^{75)}$

Two studies investigated the relationship between mortality and MCR. Among 11,867 participants from three different cohorts over a median follow-up of 28 months, MCR at baseline was associated with increased mortality overall $(\mathrm{aHR}=1.69)$ and 2 -year mortality $(\mathrm{aHR}=1.89)$ even after adjusting for gait speed and memory test scores. ${ }^{76)}$ In a study of 3,778 French women followed up for 19 years, MCR was associated with mortality at 10 years $(\mathrm{aHR}=1.27), 15$ years $(\mathrm{aHR}=1.22)$, and 19 years $(\mathrm{aHR}=1.41)^{39)}$

\section{MCR Studies in Korea}

Two studies examined the epidemiology of MCR in Korea. The MCR multi-country prevalence study included a sample of 549 individuals aged 65 to 102 years (63.8\% women) from the Korean Longitudinal Study on Health and Aging (KLoSHA). ${ }^{23)}$ The prevalence of MCR in this cohort was $10.0 \%$, compared to $8.02 \%$ in the nationwide Korean Frailty and Aging Cohort Study (KFACS) that included 2,881 community-dwelling older adults aged 70-84 years ( $52 \%$ women; mean age, 75.9 years). ${ }^{43)}$ The prevalence of MCR did not increase with age (70-74 years, $8.90 \%$; $75-79$ years, $7.06 \%$; and $80-84$ years, $8.04 \%$ ). Similar to previous studies, the MCR group had lower education levels and reduced physical activity, higher prevalence of hypertension and diabetes, and higher numbers of comorbidities and medications as compared to those in the non-MCR group. Participants with MCR had greater difficulty with respect to mobility and were more likely to report a history of falls. In addition, participants with MCR rated their health poorer compared to that in those without MCR. ${ }^{43)}$ In contrast, participants with MCR did not show significant differences in $\mathrm{BMI}$ or depressive symptoms. MCR in the KFACS cohort was associated with a decline in global cognitive function, attention, processing speed, and EF.

While these two cohort studies are not fully representative of the entire population of older Korean adults, the MCR estimates are consistent with the MCR prevalence reported in developed countries.

\section{BIOLOGY OF MCR}

Four studies reported on the relationships between brain imaging and MCR including white matter and gray matter abnormalities and brain volume and atrophy. ${ }^{66)}$ A study of 358 participants from two cohorts in France and India reported white matter hyperintensities (WMH) on magnetic resonance imaging (MRI) in $72.9 \%$ of the participants. WMH in the frontal, parieto-occipital, temporal, basal ganglia, cerebellum, or brainstem were not associated with MCR in either of the two cohorts. ${ }^{77)} \mathrm{WMH}$ are ubiquitous in aging populations and have often been regarded as non-specific. Another study of 139 participants from the Kerala-Einstein Study in India investigated the relationship between MCR and brain WMH as well as the presence of lacunar infarctions and microbleeds. ${ }^{27)}$ In this study, only the presence of lacunar infarctions in the frontal lobe was correlated with the presence of MCR at cross-section. All other parameters including WMH, other stroke, lacunar infarctions in other areas, and microbleeds were not associated with MCR. The authors assumed that vascular mechanisms other than WMH may contribute to the pathophysiology of MCR; however, they emphasized that the evaluation of WMH was based solely on manual quantification and not on automatic measurements that could provide a more objective measure and metric information such as volume. The correlation between global and regional brain volumes with MCR syndrome was evaluated in 171 participants from France. ${ }^{28)}$ Multiple logistic regression models showed that smaller volumes of total gray matter, total cortical gray matter, premotor cortex, prefrontal cortex, and dorsolateral segment of prefrontal cortex were associated with MCR. Similar to previous studies, $\mathrm{WMH}$ and total white matter volume were not correlated with MCR. Negative results were also found for hippocampus and subcortical gray matter volumes. Similar results were reported in a larger study that included 267 participants from three cohorts. ${ }^{78)}$ The significant gray matter volume covariance pattern associated with MCR even after adjusting for demographic characteristics was primarily composed of the supplementary motor, insular, and prefrontal cortex regions (Fig. 1). In contrast, relatively less atrophied regions as a function of MCR included the cerebellum as well as the inferior and middle temporal, para-hippocampal, and precuneus regions. The authors ${ }^{79)}$ concluded that MCR was primarily associated with gray matter atrophy in brain regions previously linked to the control aspects of gait such as motor planning and modulation.

The underlying biological and genetic mechanisms for MCR have not yet been established and few studies have been published to date. A study of 530 community-dwelling Ashkenazi Jewish adults age 65 years and older reported that single nucleotide polymorphisms in the transcriptional regulatory regions of cytokine interleukin 10 (IL10) were associated with the incidence of MCR over a median follow-up of 2.99 years. ${ }^{80)}$ Inflammation may play a 


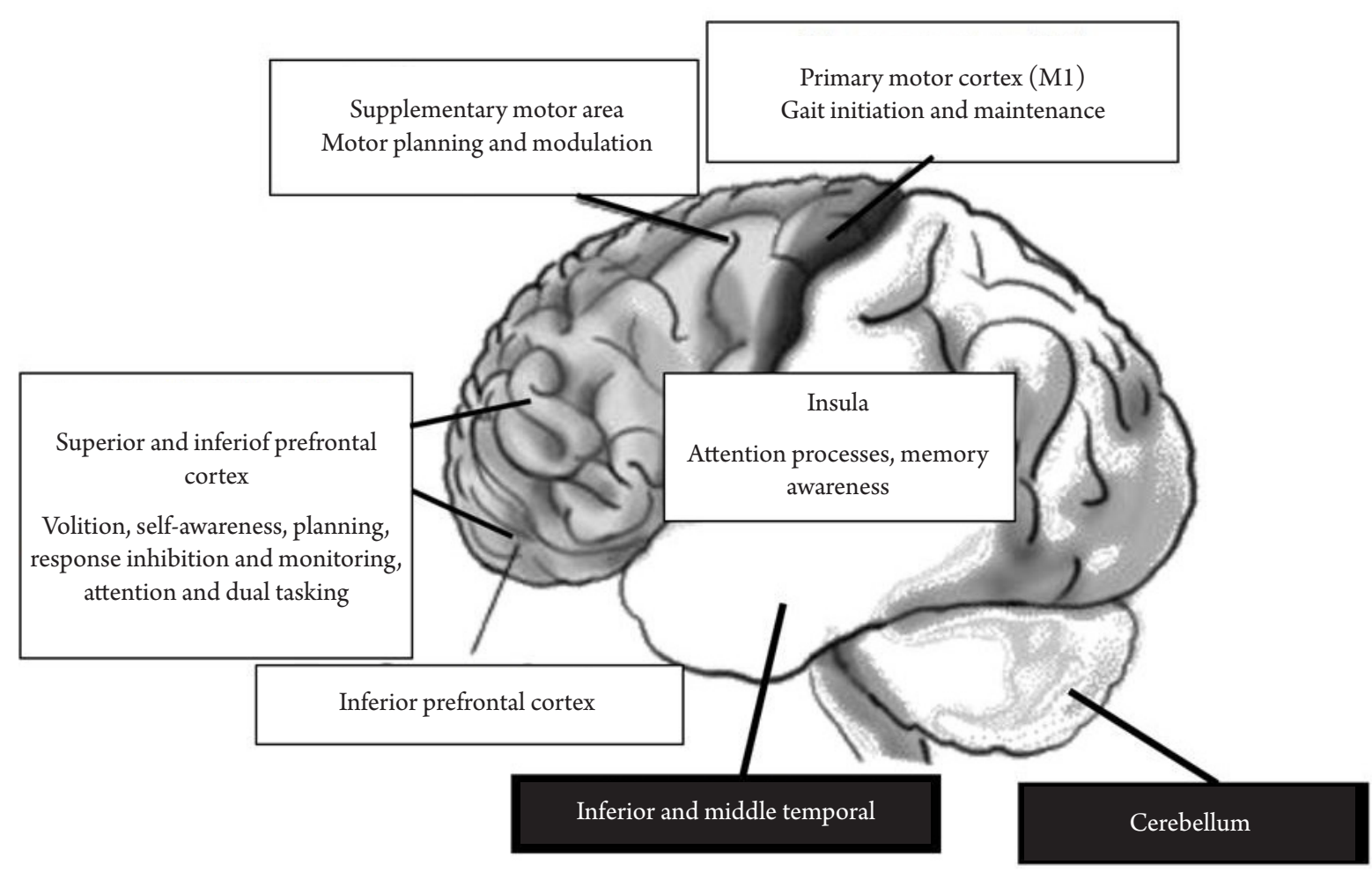

Fig. 1. Brain areas involved in motoric cognitive risk syndrome (MCR) and related cognitive mechanisms responsible for gait control (white boxes, more atrophied regions as a function of MCR; black boxes, less atrophied regions as a function of MCR).

significant role in the pathogenesis of dementia and cognitive decline; ${ }^{81)}$ this finding also suggests a role for inflammation in MCR pathogenesis. A preliminary study of the polygenic effects of selected clinical phenotypes on MCR was conducted in 4,915 individuals, age 65 years and above from the Health and Retirement Study ${ }^{79)}$ Higher polygenic scores (PGS) for BMI and waist circumference were associated with MCR and PGS of AD showed a suggestive association, while higher PGS for higher well-being was protective. The authors suggested that obesity-related genetic traits may play an important role in the development of MCR and may serve as potential therapeutic targets in dementia prevention. Fig. 2 provides a graphical representation of the suggested biological mechanisms involved in the pathogenesis of MCR and MCI that may contribute to the increased risk of dementia in these pre-dementia syndromes.

\section{POSSIBLE INTERVENTIONS TO IMPROVE FUNCTION AND PREVENT DETERIORATION IN MCR}

No studies have reported therapeutic measures such as physical exercise or cognitive training in MCR populations to improve gait and cognition and prevent deterioration to dementia. Although epidemiological studies have shown the positive effect of mainly aerobic exercise on cognitive functions in normal or demented older adults, several meta-analyses failed to confirm the cognitive protective effect of exercise in clinical trials. ${ }^{82,83)}$ In contrast, small studies have shown cognitive training programs to improve gait functions. ${ }^{84)}$ A recent meta-analysis including 10 randomized clinical trials and a total of 351 participants showed that cognitive training interventions provided a small effect on complex walking conditions requiring higher-order EFs. ${ }^{17)}$ There is a need for largescale randomized clinical trials, perhaps using multi-modal interventions, combining physical activity with cognitive stimulation or training, in MCR to improve cognitive function and mobility and prevent further deterioration towards dementia.

\section{CONCLUSION}

With the increasing aging population worldwide, the burden of cognitive disorders such as dementia is escalating. The coexistence of physical limitations and cognitive decline are common in aging adults, leading to many detrimental effects. MCR is a pre-dementia 


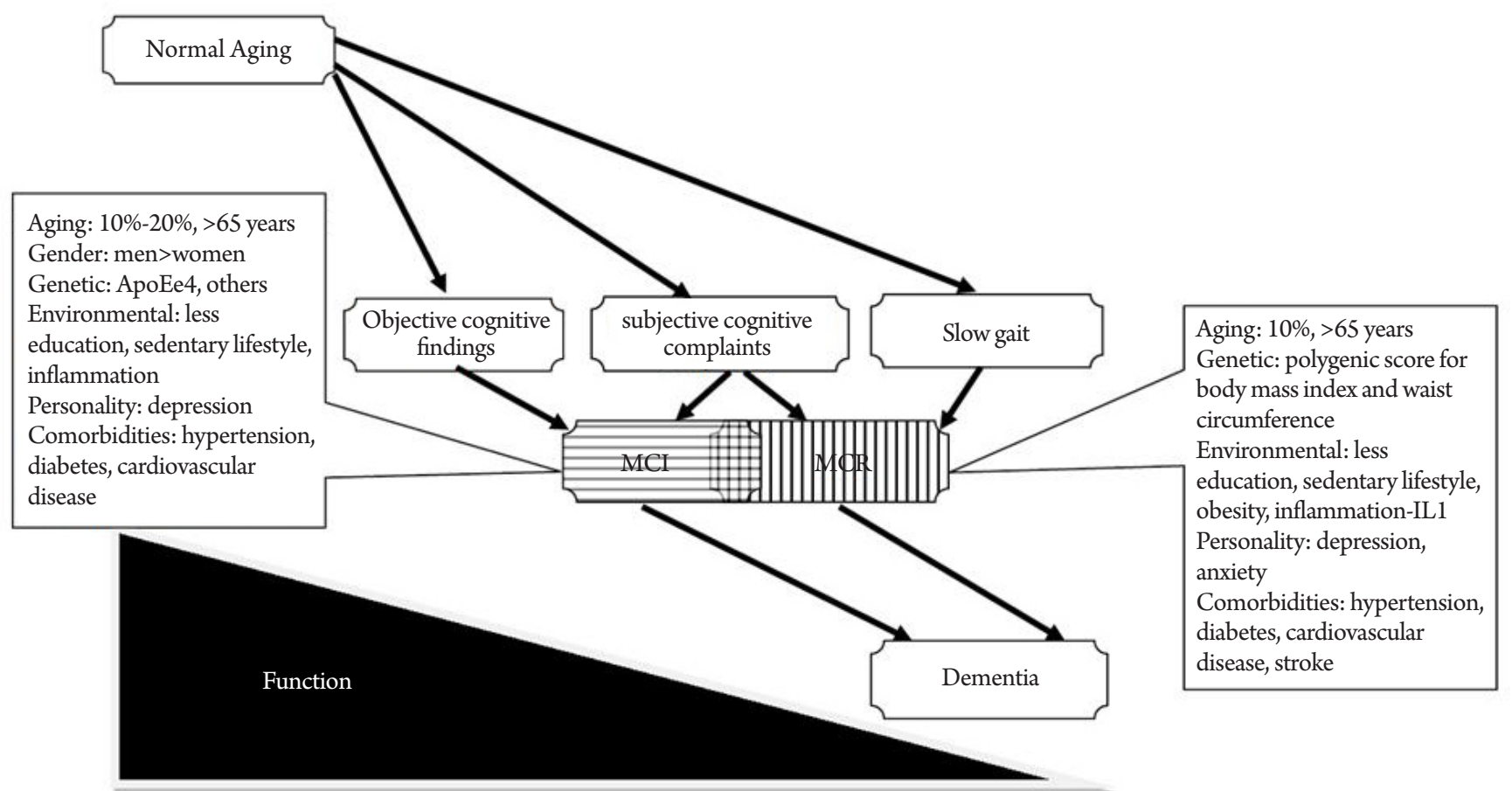

Fig. 2. Potential biological mechanisms in mild cognitive impairment (MCI) and motoric cognitive risk syndrome (MCR).

syndrome combining cognitive and motor components that increases the risk for cognitive decline and dementia as well as other age-related negative geriatric outcomes. In contrast to $\mathrm{MCI}$, the diagnosis of MCR does not rely on formal neuropsychological assessments; thus, it requires fewer resources and is independent of language and level of education. The biology of MCR remains obscure, and further studies are needed to investigate the relationship between physical and cognitive domains in older adults. The results of such studies could facilitate the design of more effective preventive intervention strategies against dementia and other deleterious effects of aging. Recent studies suggest that one in 10-12 community-residing Korean seniors may have MCR. The MCR concept is of particular importance in Korea as it is one of the most rapidly aging nations worldwide. ${ }^{85,86)}$

\section{ACKNOWLEDGEMENTS}

\section{CONFLICT OF INTEREST DISCLOSURES}

The researchers claim no conflicts of interest.

\section{FUNDING}

The research was supported by the National Institute on Aging (Grant No. R56AG057548).

\section{AUTHOR CONTRIBUTIONS}

Conceptualization, JV, ZM; Methodology, JV, EA; Data curation, ZM, EA; Project administration, EA; Writing original draft, ZM; Writing, review \& editing, JV, EA.

\section{REFERENCES}

1. United Nations Department of Economic and Social Affairs. World Population Prospects 2019: Highlights. New York, NY: United Nations; 2019.

2. Alzheimer's Disease International. World Alzheimer Report 2019: attitudes to dementia. London: Alzheimer's Disease International; 2019.

3. Verghese J, LeValley A, Hall CB, Katz MJ, Ambrose AF, Lipton RB. Epidemiology of gait disorders in community-residing older adults. J Am Geriatr Soc 2006;54:255-61.

4. Montero-Odasso M, Verghese J, Beauchet O, Hausdorff JM. Gait and cognition: a complementary approach to understanding brain function and the risk of falling. J Am Geriatr Soc 2012; 60:2127-36.

5. Cohen JA, Verghese J. Gait and dementia. Handb Clin Neurol 2019; 167:419-27.

6. Ayers E, Verghese J. Diagnosing motoric cognitive risk syndrome 
to predict progression to dementia. Neurodegener Dis Manag 2014;4:339-42.

7. Grande G, Triolo F, Nuara A, Welmer AK, Fratiglioni L, Vetrano DL. Measuring gait speed to better identify prodromal dementia. Exp Gerontol 2019;124:110625.

8. Ezzati A, Katz MJ, Lipton ML, Lipton RB, Verghese J. The association of brain structure with gait velocity in older adults: a quantitative volumetric analysis of brain MRI. Neuroradiology 2015;57:851-61.

9. Yogev-Seligmann G, Hausdorff JM, Giladi N. The role of executive function and attention in gait. Mov Disord 2008;23:329-42.

10. Holtzer R, Wang C, Lipton R, Verghese J. The protective effects of executive functions and episodic memory on gait speed decline in aging defined in the context of cognitive reserve. J Am Geriatr Soc 2012;60:2093-8.

11. Holtzer R, Verghese J, Xue X, Lipton RB. Cognitive processes related to gait velocity: results from the Einstein Aging Study. Neuropsychology 2006;20:215-23.

12. Atkinson HH, Rosano C, Simonsick EM, Williamson JD, Davis C, Ambrosius WT, et al. Cognitive function, gait speed decline, and comorbidities: the health, aging and body composition study. J Gerontol A Biol Sci Med Sci 2007;62:844-50.

13. Martin KL, Blizzard L, Wood AG, Srikanth V, Thomson R, Sanders LM, et al. Cognitive function, gait, and gait variability in older people: a population-based study. J Gerontol A Biol Sci Med Sci 2013;68:726-32.

14. Mielke MM, Roberts RO, Savica R, Cha R, Drubach DI, Christianson T, et al. Assessing the temporal relationship between cognition and gait: slow gait predicts cognitive decline in the Mayo Clinic Study of Aging. J Gerontol A Biol Sci Med Sci 2013;68:929-37.

15. Parihar R, MahoneyJR, Verghese J. Relationship of gait and cognition in the elderly. Curr Transl Geriatr Exp Gerontol Rep 2013;2:167-73.

16. Wrightson JG, Twomey R, Ross EZ, Smeeton NJ. The effect of transcranial direct current stimulation on task processing and prioritisation during dual-task gait. Exp Brain Res 2015;233: 1575-83.

17. Marusic U, Verghese J, Mahoney JR. Cognitive-based interventions to improve mobility: a systematic review and meta-analysis. J Am Med Dir Assoc 2018;19:484-491. e3.

18. Verghese J, Lipton RB, Hall CB, Kuslansky G, Katz MJ, Buschke $\mathrm{H}$. Abnormality of gait as a predictor of non-Alzheimer's dementia. N Engl J Med 2002;347:1761-8.

19. Verghese J, Holtzer R, Lipton RB, Wang C. Mobility stress test approach to predicting frailty, disability, and mortality in high-functioning older adults. J Am Geriatr Soc 2012;60:1901-5.
20. Buracchio T, Dodge HH, Howieson D, Wasserman D, Kaye J. The trajectory of gait speed preceding mild cognitive impairment. Arch Neurol 2010;67:980-6.

21. Verghese J, Wang C, Lipton RB, Holtzer R. Motoric cognitive risk syndrome and the risk of dementia. J Gerontol A Biol Sci Med Sci 2013;68:412-8.

22. Petersen RC. Mild cognitive impairment. Continuum (Minneap Minn) 2016;22(2 Dementia):404-18.

23. Verghese J, Annweiler C, Ayers E, Barzilai N, Beauchet O, Bennett DA, et al. Motoric cognitive risk syndrome: multicountry prevalence and dementia risk. Neurology 2014;83:718-26.

24. Verghese J, Ayers E, Barzilai N, Bennett DA, Buchman AS, Holtzer R, et al. Motoric cognitive risk syndrome: multicenter incidence study. Neurology 2014;83:2278-84.

25. Doi T, Verghese J, Shimada H, Makizako H, Tsutsumimoto K, Hotta R, et al. Motoric cognitive risk syndrome: prevalence and risk factors in Japanese seniors. J Am Med Dir Assoc 2015;16: 1103.e21-5.

26. Kumai K, Meguro K, Kasai M, Nakamura K, Nakatsuka M. Neuroepidemiologic and neurobehavioral characteristics of motoric cognitive risk syndrome in an old-old population: the Kurihara project. Dement Geriatr Cogn Dis Extra 2016;6:176-82.

27. Wang N, Allali G, Kesavadas C, Noone ML, Pradeep VG, Blumen HM, et al. Cerebral small vessel disease and motoric cognitive risk syndrome: results from the Kerala-Einstein Study. J Alzheimers Dis 2016;50:699-707.

28. Beauchet O, Allali G, Annweiler C, Verghese J. Association of motoric cognitive risk syndrome with brain volumes: results from the GAIT study. J Gerontol A Biol Sci Med Sci 2016; 71:1081-8.

29. Allali G, Ayers EI, Verghese J. Motoric cognitive risk syndrome subtypes and cognitive profiles. J Gerontol A Biol Sci Med Sci 2016;71:378-84.

30. Doi T, Shimada H, Makizako H, Tsutsumimoto K, Verghese J, Suzuki T. Motoric cognitive risk syndrome: association with incident dementia and disability. J Alzheimers Dis 2017;59:77-84.

31. Sekhon H, Allali G, Launay CP, Chabot J, Beauchet O. The spectrum of pre-dementia stages: cognitive profile of motoric cognitive risk syndrome and relationship with mild cognitive impairment. Eur J Neurol 2017;24:1047-54.

32. Beauchet O, Sekhon H, Barden J, Liu-Ambrose T, Chester VL, Szturm T, et al. Association of motoric cognitive risk syndrome with cardiovascular disease and risk factors: results from an original study and meta-analysis. J Alzheimers Dis 2018;64:875-87.

33. Maguire FJ, Killane I, Creagh AP, Donoghue O, Kenny RA, Reilly $\mathrm{RB}$. Baseline association of motoric cognitive risk syndrome with sustained attention, memory, and global cognition. J Am 
Med Dir Assoc 2018;19:53-8.

34. Chhetri JK, Han C, Dan X, Ma L, Chan P. Motoric cognitive risk syndrome in a Chinese older adult population: prevalence and associated factors. J Am Med Dir Assoc 2020;21:136-7.

35. Aguilar-Navarro SG, Mimenza-Alvarado AJ, Aguilar-Esquivel JE, Yeverino-Castro SG, Juarez-Cedillo T, Mejia-Arango S. Motoric Cognitive risk syndrome: prevalence and risk of cognitive impairment in a population studied in the Mexican Health and Aging Study 2012-2015. J Nutr Health Aging 2019;23:227-31.

36. Lau H, Mat Ludin AF, Shahar S, Badrasawi M, Clark BC. Factors associated with motoric cognitive risk syndrome among low-income older adults in Malaysia. BMC Public Health 2019; 19(Suppl 4):462.

37. Beauchet O, Sekhon H, Schott AM, Rolland Y, Muir-Hunter S, Markle-Reid M, et al. Motoric cognitive risk syndrome and risk for falls, their recurrence, and postfall fractures: results from a prospective observational population-based cohort study. J Am Med Dir Assoc 2019;20:1268-73.

38. Beauchet O, Sekhon H, Launay CP, Rolland Y, Schott AM, Allali $\mathrm{G}$. Motoric cognitive risk syndrome and incident dementia: results from a population-based prospective and observational cohort study. Eur J Neurol 2020;27:468-74.

39. Beauchet O, Sekhon H, Launay CP, Chabot J, Rolland Y, Schott $\mathrm{AM}$, et al. Motoric cognitive risk syndrome and mortality: results from the EPIDOS cohort. Eur J Neurol 2019;26:794-e56.

40. Sekhon H, Allali G, Beauchet O. The association of anxio-depressive disorders and depression with motoric cognitive risk syndrome: results from the baseline assessment of the Canadian longitudinal study on aging. Geroscience 2019;41:409-18.

41. Sekhon H, Allali G, Beauchet O. Motoric cognitive risk syndrome and cardiovascular diseases and risk factors in the Canadian population: results from the baseline assessment of the Canadian longitudinal study on aging. Arch Gerontol Geriatr 2019; 85:103932.

42. Verghese J, Wang C, Bennett DA, Lipton RB, Katz MJ, Ayers E. Motoric cognitive risk syndrome and predictors of transition to dementia: a multicenter study. Alzheimers Dement 2019;15: 870-7.

43. Shim H, Kim M, Won CW. Motoric cognitive risk syndrome is associated with processing speed and executive function, but not delayed free recall memory: the Korean Frailty and Aging Cohort Study (KFACS). Arch Gerontol Geriatr 2020;87:103990.

44. Morris JC, Heyman A, Mohs RC, Hughes JP, van Belle G, Fillenbaum G, et al. The Consortium to Establish a Registry for Alzheimer's Disease (CERAD). Part I. Clinical and neuropsychological assessment of Alzheimer's disease. Neurology 1989; 39:1159-65.
45. Pfeiffer E. A short portable mental status questionnaire for the assessment of organic brain deficit in elderly patients. J Am Geriatr Soc 1975;23:433-41.

46. Hughes CP, Berg L, Danziger WL, Coben LA, Martin RL. A new clinical scale for the staging of dementia. Br J Psychiatry 1982; 140:566-72.

47. Larner AJ. AD8 informant questionnaire for cognitive impairment: pragmatic diagnostic test accuracy study. J Geriatr Psychiatry Neurol 2015;28:198-202.

48. Morris JC. The Clinical Dementia Rating (CDR): current version and scoring rules. Neurology 1993;43:2412-4.

49. van Uden CJ, Besser MP. Test-retest reliability of temporal and spatial gait characteristics measured with an instrumented walkway system (GAITRite). BMC Musculoskelet Disord 2004; $5: 13$.

50. Capistrant BD, Glymour MM, Berkman LF. Assessing mobility difficulties for cross-national comparisons: results from the World Health Organization Study on Global AGEing and Adult Health.J Am Geriatr Soc 2014;62:329-35.

51. Sekhon H, Launay CP, Chabot J, Allali G, Beauchet O. Motoric cognitive risk syndrome: could it be defined through increased five-times-sit-to-stand test time, rather than slow walking speed? Front Aging Neurosci 2019; 10:434.

52. Ayers E, Verghese J. Gait dysfunction in motoric cognitive risk syndrome. J Alzheimers Dis 2019;71(s1):S95-S103.

53. Katz S, Ford AB, Moskowitz RW, Jackson BA, Jaffe MW. Studies of illness in the aged: the index of ADL: a standardized measure of biological and psychosocial function. JAMA 1963;185:914-9.

54. American Psychiatric Association. Diagnostic and statistical manual of mental disorders. 4th ed. Washington, DC: American Psychiatric Association; 1994.

55. Folstein MF, Folstein SE, McHugh PR. "Mini-mental state”. A practical method for grading the cognitive state of patients for the clinician. J Psychiatr Res 1975;12:189-98.

56. Sharp ES, Gatz M. Relationship between education and dementia: an updated systematic review. Alzheimer Dis Assoc Disord 2011;25:289-304.

57. Stephen R, Hongisto K, Solomon A, Lonnroos E. Physical activity and Alzheimer's disease: a systematic review. J Gerontol A Biol Sci Med Sci 2017;72:733-9.

58. Sofi F, Valecchi D, Bacci D, Abbate R, Gensini GF, Casini A, et al. Physical activity and risk of cognitive decline: a meta-analysis of prospective studies. J Intern Med 2011;269:107-17.

59. Verghese J, Wang C, Xue X, Holtzer R. Self-reported difficulty in climbing up or down stairs in nondisabled elderly. Arch Phys Med Rehabil 2008;89:100-4.

60. Rovio S, Spulber G, Nieminen LJ, Niskanen E, Winblad B, Tu- 
omilehto J, et al. The effect of midlife physical activity on structural brain changes in the elderly. Neurobiol Aging 2010;31: 1927-36.

61. Kivipelto M, Helkala EL, Laakso MP, Hanninen T, Hallikainen M, Alhainen K, et al. Midlife vascular risk factors and Alzheimer's disease in later life: longitudinal, population based study. BMJ 2001;322:1447-51.

62. Gorelick PB, Scuteri A, Black SE, Decarli C, Greenberg SM, Iadecola C, et al. Vascular contributions to cognitive impairment and dementia: a statement for healthcare professionals from the American Heart Association/American Stroke Association. Stroke 2011;42:2672-713.

63. Iadecola C, Yaffe K, Biller J, Bratzke LC, Faraci FM, Gorelick PB, et al. Impact of hypertension on cognitive function: a scientific statement from the American Heart Association. Hypertension 2016;68:e67-e94.

64. Kristensen RU, Norgaard A, Jensen-Dahm C, Gasse C, Wimberley T, Waldemar G. Polypharmacy and potentially inappropriate medication in people with dementia: a nationwide study. J Alzheimers Dis 2018;63:383-94.

65. Panza F, Frisardi V, Capurso C, D’Introno A, Colacicco AM, Imbimbo BP, et al. Late-life depression, mild cognitive impairment, and dementia: possible continuum? Am J Geriatr Psychiatry 2010;18:98-116.

66. Sekhon H, Allali G, Launay CP, Barden J, Szturm T, Liu-Ambrose T, et al. Motoric cognitive risk syndrome, incident cognitive impairment and morphological brain abnormalities: systematic review and meta-analysis. Maturitas 2019;123:45-54.

67. Wechsler D. Wechsler memory scale-revised: manual. San Antonio, TX: Psychological Corporation; 1987.

68. Albert MS, DeKosky ST, Dickson D, Dubois B, Feldman HH, Fox NC, et al. The diagnosis of mild cognitive impairment due to Alzheimer's disease: recommendations from the National Institute on Aging-Alzheimer's Association workgroups on diagnostic guidelines for Alzheimer's disease. Alzheimers Dement 2011;7:270-9.

69. Chhetri JK, Chan P, Vellas B, Cesari M. Motoric cognitive risk syndrome: predictor of dementia and age-related negative outcomes. Front Med (Lausanne) 2017;4:166.

70. Pfortmueller CA, Lindner G, Exadaktylos AK. Reducing fall risk in the elderly: risk factors and fall prevention, a systematic review. Minerva Med 2014;105:275-81.

71. Tinetti ME, Williams CS. Falls, injuries due to falls, and the risk of admission to a nursing home. N Engl J Med 1997;337:127984.
72. Callisaya ML, Ayers E, Barzilai N, Ferrucci L, Guralnik JM, Lipton RB, et al. Motoric cognitive risk syndrome and falls risk: a multi-center study. J Alzheimers Dis 2016;53:1043-52.

73. Allali G, Verghese J. Management of gait changes and fall risk in MCI and dementia. Curr Treat Options Neurol 2017;19:29.

74. Clegg A, Young J, Iliffe S, Rikkert MO, Rockwood K. Frailty in elderly people. Lancet 2013;381:752-62.

75. Sathyan S, Ayers E, Gao T, Milman S, Barzilai N, Rockwood K, et al. Frailty and risk of incident motoric cognitive risk syndrome. J Alzheimers Dis 2019;71(s1):S85-S93.

76. Ayers E, Verghese J. Motoric cognitive risk syndrome and risk of mortality in older adults. Alzheimers Dement 2016;12:556-64.

77. Mergeche JL, Verghese J, Allali G, Wang C, Beauchet O, Kumar VG, et al. White matter hyperintensities in older adults and motoric cognitive risk syndrome. J Neuroimaging Psychiatry Neurol 2016;1:73-8.

78. Blumen HM, Allali G, Beauchet O, Lipton RB, Verghese J. A gray matter volume covariance network associated with the motoric cognitive risk syndrome: a multicohort MRI study.J Gerontol A Biol Sci Med Sci 2019;74:884-9.

79. Sathyan S, Wang T, Ayers E, Verghese J. Genetic basis of motoric cognitive risk syndrome in the Health and Retirement Study. Neurology 2019;92:e1427-e1434.

80. Sathyan S, Barzilai N, Atzmon G, Milman S, Ayers E, Verghese J. Association of anti-inflammatory cytokine IL10 polymorphisms with motoric cognitive risk syndrome in an Ashkenazi Jewish population. Neurobiol Aging 2017;58:238.e1-238.e8.

81. Heneka MT, Carson MJ, El Khoury J, Landreth GE, Brosseron F, Feinstein DL, et al. Neuroinflammation in Alzheimer's disease. Lancet Neurol 2015;14:388-405.

82. Young J, Angevaren M, Rusted J, Tabet N. Aerobic exercise to improve cognitive function in older people without known cognitive impairment. Cochrane Database Syst Rev 2015;(4): CD005381.

83. Forbes D, Forbes SC, Blake CM, Thiessen EJ, Forbes S. Exercise programs for people with dementia. Cochrane Database Syst Rev 2015;(4):CD006489.

84. Verghese J, Mahoney J, Ambrose AF, Wang C, Holtzer R. Effect of cognitive remediation on gait in sedentary seniors. J Gerontol A Biol Sci Med Sci 2010;65:1338-43.

85. Kim J, Lee Y, Won CW, Lee KE, Chon D. Nutritional status and frailty in community-dwelling older Korean adults: the Korean Frailty and Aging Cohort Study. J Nutr Health Aging 2018; 22:774-8.

86. Statistics Korea. Statistics of the elderly in 2013. Daejeon, Korea: Statistics Korea; 2014. 